\title{
Cartesian Task Allocation for Cooperative, Multilateral Teleoperation under Time Delay
}

\author{
Michael Panzirsch ${ }^{1}$, Ribin Balachandran ${ }^{1}$ and Jordi Artigas ${ }^{1}$
}

\begin{abstract}
Field robots - robots used in unstructured and dynamic environments - and teleoperation have shifted into the focus of a variety of industrial branches in the past few years. The lack of space in the atomic industry, on oil platforms and in space applications demands additional adaptations to current robotic setups. In this paper a MMSS (Multi-Master-SingleSlave) haptic teleoperation system is proposed through which one operator using two master arms can manipulate objects in a cooperative way via one slave robot and a virtual gripping point. To ease the execution of a peg-in-hole task of big objects, a task allocation in the cartesian frame of the virtual gripping point is introduced additionally. The stability of this multilateral system with time delay is guaranteed by the Time Domain Passivity Approach. Therefore the system is divided into several modular subsystems which renders the system easily adaptable to other scenarios.
\end{abstract}

Index Terms-cooperation, multilateral teleoperation, task allocation, peg-in-hole, MOMR, MMMS, TDPA

\section{INTRODUCTION}

With the progress of robotic technology, the areas of its application emerged immensely in the past. It became feasible to use robots for plant maintenance or complex constructional tasks in hazardous environments (see [1]). Still, efficiency and ergonomics are key factors for success, especially when robots in unstructured, dynamic and narrow environments need to be teleoperated from distance.

Research on Multi-Master-Multi-Slave systems (MMMS) has mainly focused on interactions via objects (environmental impedances more generally, [2],[3]), model-mediated cooperative teleoperation and collision avoidance ([4],[5]). Those challenges are crucial e.g. in minimally invasive surgery, whereat efficiency and safety can be improved through the use of MMMS systems (compare [6]).

The above mentioned MMMS systems are mostly based on a bilateral control rationale, in which the haptic information is only exchanged between a single master and a single slave system ([7]). Other works propose the coupling of two master devices to one slave robot, rendering a quasi-trilateral system ([8], [3]). [9],[10] propose true trilateral systems, where the involved master devices are also coupled to each other. Those systems are so far preferentially used for training and rehabilitation aspects rather than for cooperation.

In those multilateral systems, a variety of task allocation types were proposed. In [11], one master controlled the endeffector of the slave robot, whereas a second master was used to change the configuration of the redundant slave robot

\footnotetext{
${ }^{1}$ Michael Panzirsch, Ribin Balachandran and Jordi Artigas are with the department for Analysis and Control of Advanced Robotic Systems , German Aerospace Center, Oberpfaffenhofen, Germany michael.panzirsch@dlr.de
}

in a way that the end-effector pose is not affected. In [12], projective force mappings were introduced to impose specific boundaries onto the slave robot's motion. As adaptive control has been applied, models of hardware, operator and environment were required. In [13], two masters with different degrees of freedom (DoF) were used to control a slave robot with three degrees of freedom. Haptic feedback could constitutionally only be displayed on the available DoFs of each master device. Lee et al. showed in [1] that through the Time Domain Passivity Approach (TDPA), a disjoint axis control with time delay is feasible, such that two subsets of DoFs of a redundant slave robot - consisting of a mobile platform moving a serial robot - could be independently controlled by two master devices. A similar setup has been applied in [14].

Also the approaches [10] and [15] on multilateral systems are based on the TDPA. Time delay in MMMS systems is handled in [9] and [16] by the wave variable method. In [17], the effect of time delay on different types of cooperative control methods in MMMS systems is analyzed more closely. [18] and [19] developed approaches for SMMS (SingleMaster-Multi-Slave) systems able to handle big objects despite delayed communication. In both papers a local grasping controller organized the gripping of a big object by the slave robots. In [18], the scattering transform was used to consider the delay. [19] proposed a model-based adaptive synchronizing controller with damping injection term.

Clearly, the dexterity levels of an operator who is performing a telemanipulation is influenced by the telerobotic platform being used. The methods presented in this paper aim at increasing the skillfulness of an operator in manipulating large objects through the distance in unstructured and especially narrow environments allowing only compact robotic systems. The addressed scenario consists of a teleoperation system with two masters and a single slave and a constrained communication channel with time delay. To demonstrate the efficiency of the proposed method, a peg-in-hole task of a long pipe has been used. The system is designed in a multilateral fashion, that is, all the involved robots are coupled with each other, allowing an accurate and intuitive interaction. A task allocation in the Cartesian space is furthermore introduced to ease the peg-in-hole process.

This paper is structured as follows: Chapter II introduces the multilateral structure and the considered control approach. In chapter III, the scenario of cooperation is explained more closely and the required extensions of the system are implemented. The conducted experiments are presented in chapter IV and the results are summed up in chapter V. 


\section{Multilateral Control}

The system design is based on the multilateral control approach proposed in [10]. A generic so-called track-POPC system based on the TDPA has been introduced which allows a variable and guaranteed stable haptic interaction of an arbitrary number of agents (see Fig. 1). Agents can be human operators with master devices, slaves in their environment or autonomous intelligent units. Each track represents the bilateral haptic connection between two agents. Fig. 2 depicts

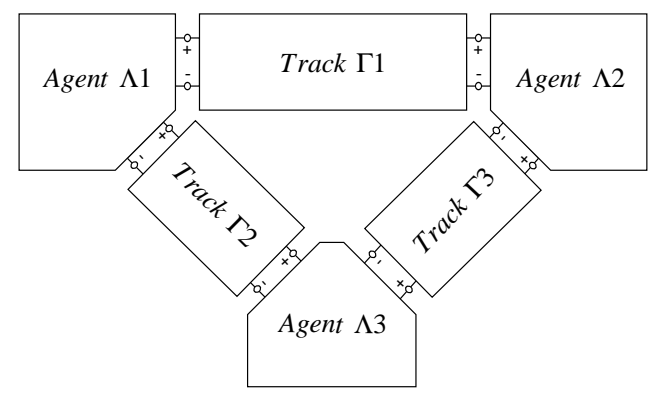

Fig. 1. Exemplary assembly of Tracks to a multilateral system

the signal flow diagram of a Position-Position-architecture (PP) with time delay. On both sides of that communication channel, a PI-controller is located which acts like a virtual spring-damper system on the master and slave positions and velocities respectively. In order to use the passivitybased TDPA, the energy behavior of the system has to be evaluated. Therefore the bilateral signal flow diagram has to be transduced into the network representation (Fig. 3) - the electrical analog of the signal flow diagram - such that energies can be measured at the subsystem's ports. This transformation is explained in [20]. The time delays $T 1$ and $T 2$ in the communication channels are represented by the Time Delay Power Networks TDPN (proposed in [21]). The agents contain besides human, environment and the hardware devices also the power distributing subsystems PCU that have been introduced in [10]. The energy that can be measured at

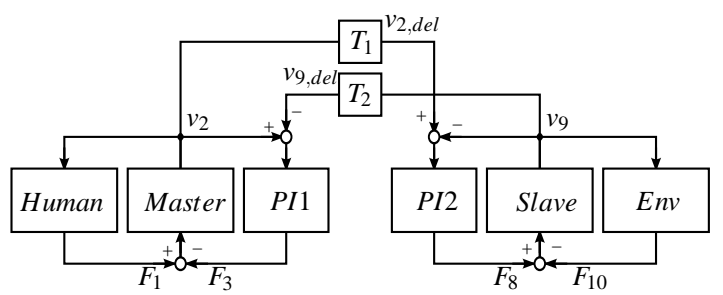

Fig. 2. Signal Flow Diagram of a teleoperation system with position-position architecture

the ports of the network subsystems is considered to check the passivity of e.g. a 2-port subsystem (see Fig. 4):

$$
E^{L, 2 P}(t)+E^{R, 2 P}(t) \geq 0
$$

where $E^{R / L, 2 P}$ are the energies flowing on the right/left side of the 2-port $(2 P)$ which can be computed based on conjugate power pairs at each port. The power $P^{L / R, 2 P}$ flowing at the

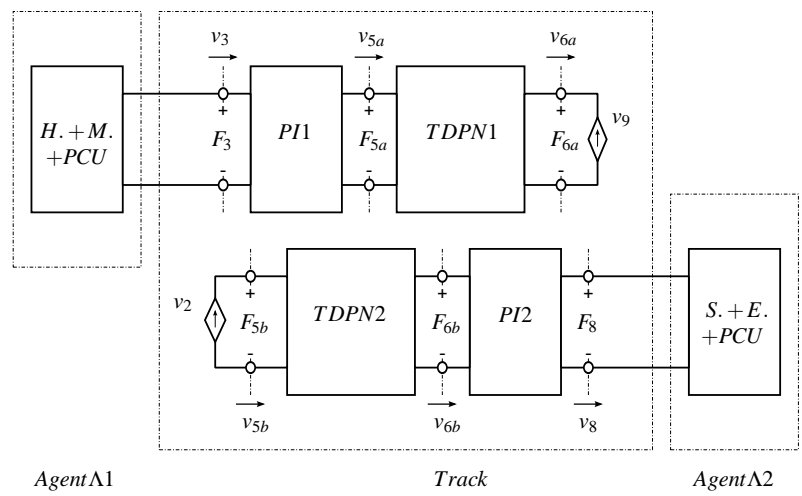

Fig. 3. Network representation of a teleoperation system with position-position architecture

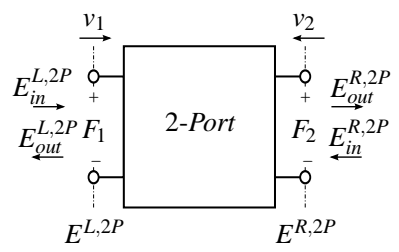

Fig. 4. In and out energies of a 2-port network

left/right port of a 2-port can be calculated as follows:

$$
P^{L, 2 P}(t)=v_{1}(t) F_{1}(t), \quad P^{R, 2 P}(t)=v_{2}(t) F_{2}(t) .
$$

Analyzing the sign of $P^{L / R, 2 P}$, one can split up the power concerning the flow directions: $P_{\text {in } / \text { out }}^{L / R, 2 P}$. For instance:

$$
\begin{aligned}
& P_{\text {in }}^{L, 2 P}(t)=\left\{\begin{array}{cc}
0, & \text { if } P^{L, 2 P}(t)<0 \\
P^{L, 2 P}(t), & \text { if } P^{L, 2 P}(t)>0
\end{array}\right. \text { and } \\
& P_{\text {out }}^{L, 2 P}(t)=\left\{\begin{array}{cc}
0, & \text { if } P^{L, 2 P}(t)>0 \\
-P^{L, 2 P}(t), & \text { if } P^{L, 2 P}(t)<0 .
\end{array}\right.
\end{aligned}
$$

The subindices in and out indicate the power (or energy) flowing into and out of the network on the left $(L)$ or the right $(R)$ side respectively. Then the in/out flowing energies $E_{\text {in/out }}^{L / R, 2 P}$ on the left/right side can be calculated:

$$
E_{\text {in } / \text { out }}^{L, 2 P}(t)=\int_{0}^{t} P_{\text {in/out }}^{L, 2 P}(\tau) d \tau, \quad E_{\text {in } / \text { out }}^{R, 2 P}(t)=\int_{0}^{t} P_{\text {in/out }}^{R, 2 P}(\tau) d \tau .
$$

As these energies can only be observed in the two directions of a track separately, the passivity condition (1) has to be reformulated:

$$
\begin{aligned}
E^{L, 2 P}(t)+ & E^{R, 2 P}(t)=E_{\text {in }}^{L, 2 P}\left(t-T_{1}\right) \\
& -E_{\text {out }}^{R, 2 P}(t)+E_{\text {in }}^{R, 2 P}\left(t-T_{2}\right)-E_{\text {out }}^{L, 2 P}(t) .
\end{aligned}
$$

Since the energies are purely increasing the approach meets with equation (2) and the conditions (3) the passivity criterion (1).

$$
E_{\text {in }}^{L, 2 P}(t)-E_{\text {out }}^{R, 2 P}(t) \geq 0, \quad E_{\text {in }}^{R, 2 P}(t)-E_{\text {out }}^{L, 2 P}(t) \geq 0
$$

In a track-POPC system two Passivity Controllers (PC) are introduced in the tracks which dissipate the energy generated in the respective PI and TDPN. In [10], it was shown 
that through the use of track-POPCs - and under the wellaccepted assumption that the agents behave passively in their interaction - the whole system is passive and thus stable. Thus, any combination of tracks and agents is proven to be stable. No models of the hardware and no further analytical stability proof is necessary. This structure and the stability control approach respectively can be easily extended to the 6-DoF case if the position controller is designed in the cartesian space such that all DoFs can be handled separately. Still, as we focus in this paper on the manipulation of big objects and on the task allocation in the multi-DoF case, new modules have to be developed and investigated concerning their energetic behavior.

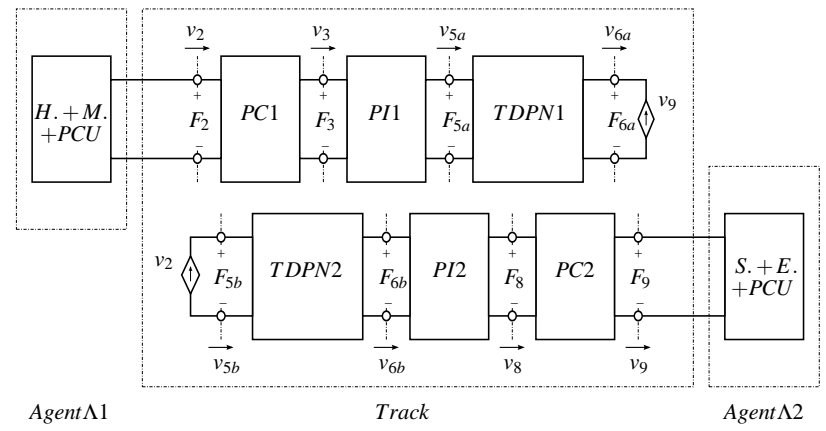

Fig. 5. Network representation of a track-POPC system with positionposition architecture

\section{Multilateral CoOperation}

The scenario of interest is a MMSS system with task allocation which should simplify the assembly of a long pipe into a plug fixed on the wall.

In a general bilateral teleoperation setup, it is tough to fulfill this task especially if the gripping position is deviating from the pipe end which has to be plugged. This complexity is visualized in Fig. 6 where the tool frames $I$ of the slave and II of the master device are coupled by track $\Gamma 1$, visualized by a spring damper system. When the operator rotates the pipe around frame $I$ into the right orientation (perpendicular to the wall) the pipe end will move downward and away from the plug position. It is difficult to keep the pipe end at the plug's position since the orientations and translations therefore need to be adapted in an iterative manner.

For such types of tasks it is helpful to introduce another master through which the same operator can control the position of an additional point on the object. In Fig. 7, master 2 is gripping at the virtual gripping point $P E$ in the pipe end. The tool center point $I I$ of master 1 determines the position of $P E$ through a projection of $I I$ via $\mathbf{T}$ onto $I I_{P R}$. Through Track $\Gamma 2$ the two devices are thus coupled in a virtual pipe like behavior. Fig. 8 depicts the aspired multilateral cooperation setup. The virtual gripping point at the pipe end $P E$ can be considered as to be manipulated by a virtual slave. The tool frames $I$ and $I I$ are projected into the respective pipe end frames $I_{P R}$ and $I_{P R}$. $I_{P R}$ represents the pose of the real pipe end. The devices are connected to

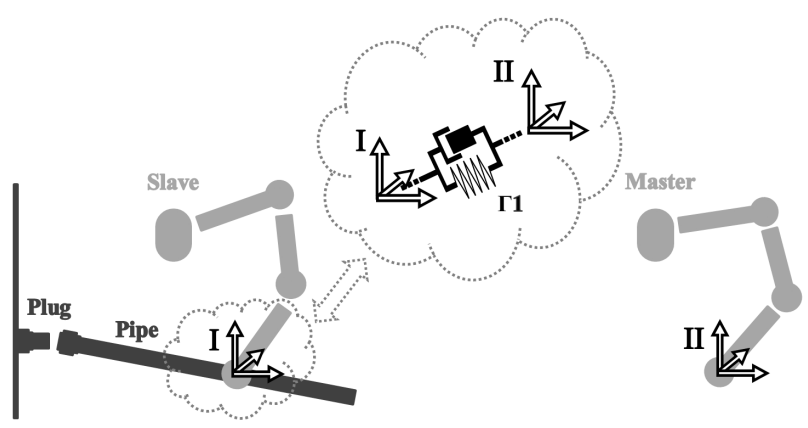

Fig. 6. Bilateral telemanipulation of a pipe

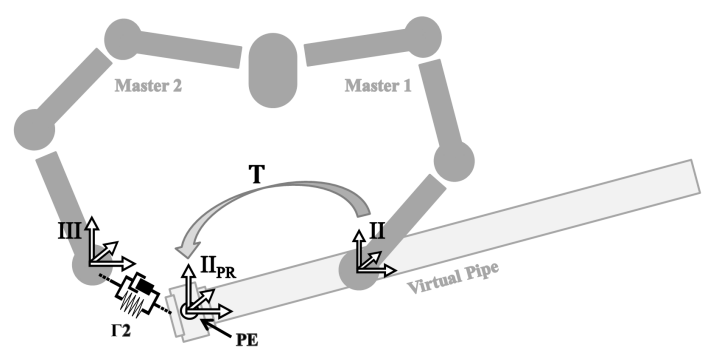

Fig. 7. Coupling of master devices through virtual pipe

each other by the tracks $\Gamma i$ in the pipe end, i.e. the track $\Gamma 1$ of Fig. 6 is projected into the pipe end. It is important

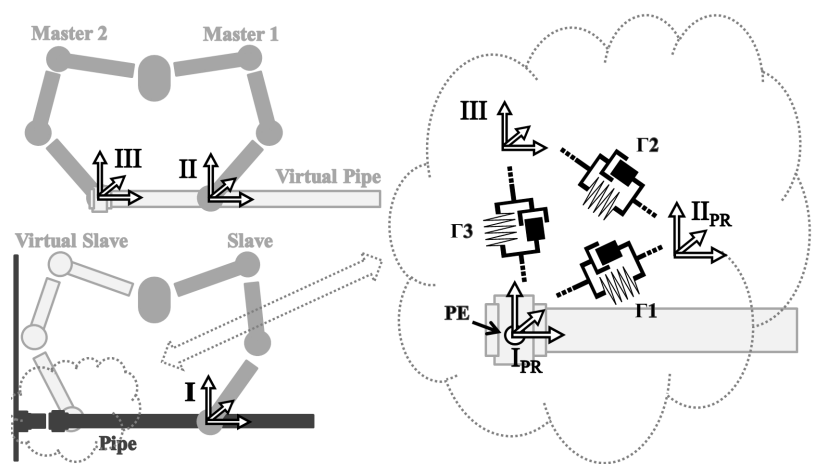

Fig. 8. Multilateral Cooperation with spatial spring in the pipe's end

that the controllers are designed in the pipe end as the task allocation will have to be implemented in this coordinate frame later on. During the plugging of the pipe the master arm controlling the pipe end should have the whole might on the pipe end's translation. Thus, the operator's right arm (master 2) can easily fix the pipe end's position close to the plug position. The task of the left operator arm (master 1) is to bring the pipe into the correct orientation - perpendicular to the wall. Therefore the Master 1 receives up to the whole authority on the rotation of the pipe end. 


\section{A. Virtual Gripping Point}

The projection of the coordinate frames $I$ and $I I$ into $I_{P R}$ and $I I_{P R}$ can be computed as follows:

$$
\begin{aligned}
& \mathbf{H}^{I_{P R} / I I_{P R}}=\mathbf{H}^{I / I I} \mathbf{T}, \mathbf{W}^{I / I I}={ }^{\mathbf{W}} \mathbf{T}^{T} \mathbf{W}^{I_{P R} / I I_{P R}} \text {, with } \\
& \mathbf{T}=\left[\begin{array}{cc}
\mathbf{T} \mathbf{R} & \mathbf{T} \mathbf{p} \\
0 & 1
\end{array}\right] \text { and } \mathbf{W}_{\mathbf{T}}=\left[\begin{array}{cc}
\mathbf{T} \mathbf{R} & \mathbf{0} \\
\mathbf{0} & \mathbf{T} \mathbf{R}
\end{array}\right]
\end{aligned}
$$

where $\mathbf{H}^{f r}$ is the homogenous transform from base frame to frame $f r$. $\mathbf{W}^{I_{P R}}$ and $\mathbf{W}^{I I_{P R}}$ are the force $F$ and torque $M$ outputs of the PI controllers which are sent to the master and slave devices ( $\mathbf{W}^{I}$ to slave, $\mathbf{W}^{I I}$ to master 1 and $\mathbf{W}^{I I I}$ to master 2). The projections of the coordinate frame $I$ to $I_{P R}$ and vice versa are exemplary depicted in Fig. 9. The transformation matrix $\mathbf{T}$ from tool frame to pipe end frame is assumed to be known. The passivity of the projection can be easily proven analytically. To guarantee the energy preservation of the fixed coupling

$$
\sum_{i=1}^{n}\left(W_{i}^{A} \dot{q}_{i}^{A}\right)=\sum_{i=1}^{n}\left(W_{i}^{B} \dot{q}_{i}^{B}\right)
$$

with the generalized velocities $\dot{q}$, has to hold. As velocities and forces/torques have to be investigated, the translational deviation $\mathbf{T} \mathbf{p}$ doesn't need to be considered. With

$$
W_{i}^{A}=\sum_{j=1}^{n}\left(\mathbf{W}_{T_{j i}} W_{j}^{B}\right) \text { and } \dot{q}_{i}^{B}=\sum_{j=1}^{n}\left(\mathbf{W}_{T_{i j}} \dot{q}_{j}^{A}\right)
$$

it can be shown that (4) is always guaranteed:

$$
\sum_{i=1}^{n}\left(\sum_{j=1}^{n}\left({ }^{\mathbf{w}_{1}} T_{j i} W_{j}^{B}\right) \dot{q}_{i}^{A}\right)=\sum_{i=1}^{n}\left(W_{i}^{B} \sum_{j=1}^{n}\left(\mathbf{w} T_{i j} \dot{q}_{j}^{A}\right)\right) .
$$

\section{B. Task Allocation}

Apart from the projection of positions and forces/torques, the task allocation has to be investigated. The task allocation factor $\alpha_{R o t / T r a n s}^{\Gamma i, L 2 R / R 2 L}$ scale the forces (Trans) and the torques (Rot) sent from the PI-controller PI2 from left to right $(L 2 R)$ or $P I 1$ from right to left $(R 2 L)$ in track $\Gamma i$ (compare Fig.2). The functionality of the task allocation is described by the intensity of the coordinate frames in Fig. 10. The arrows indicate the forces and torques sent from one device to the two others. The coordinate frame of the slave is dark as its feedback to the masters is not varied by the allocation factor $\alpha_{\text {Trans } / \text { Rot }}^{\Gamma 1, R 2 L}$ and $\alpha_{\text {Trans } / \text { Rot }}^{\Gamma 3, R 2 L}$ (see TABLE I). The translational feedback of master 2 is not altered by the task allocation $\left(\alpha_{\text {Trans }}^{\Gamma 2, R 2 L / \Gamma 3, L 2 R}=1\right)$. The translational DoFs of master 1 and the rotational DoFs of master 2 are lighter as the feedback of those DoFs is scaled down by the task allocation $\left(\alpha_{\text {Trans }}^{\Gamma 1, L 2 R / \Gamma 2, R 2 L}<1\right.$ and $\left.\alpha_{R o t}^{\Gamma 2, R 2 L / \Gamma 3, L 2 R}<1\right)$. The translations of master 2 can be affected by cross couplings if the device's orientations would be absolutely dominated by master 1 . Therefore the scalings $\alpha_{\text {Rot }}$ of master 1 and 2 should both be set to 0.5 . Fig. 11 depicts the resulting track-POPC for delayed cooperative teleoperation with virtual gripping point projections and task allocation. As the projection blocks PRi and the PC-controlled parts of the track are proven to be passive, the whole system will always be passive and thus stable.

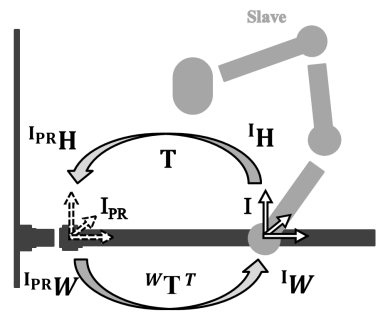

Fig. 9. Transformation of forces/torques and poses

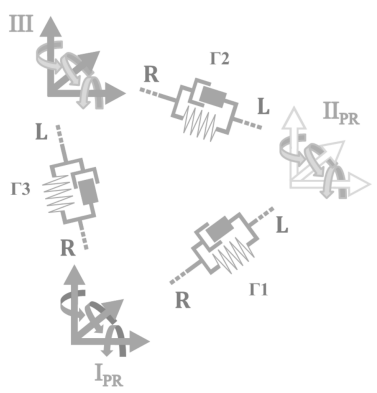

Fig. 10. Cartesian Task Allocation in the pipe end

\begin{tabular}{|c||c|c|c|}
\hline Device & \multicolumn{2}{|c|}{$\alpha$} & Value \\
\hline \hline Master 1 & $\alpha_{\text {Trans }}^{\Gamma 1, L 2 R}$ & $\alpha_{\text {Trans }}^{\Gamma 2, L 2 R}$ & 0 \\
\hline Master 1 & $\alpha_{\text {Rot }}^{I 1, L 2 R}$ & $\alpha_{\text {Rot }}^{\Gamma 2, L 2 R}$ & 0.5 \\
\hline Master 2 & $\alpha_{\text {Trans }}^{I 2, R 2 L}$ & $\alpha_{\text {Trans }}^{\Gamma 3, L 2 R}$ & 1 \\
\hline Master 2 & $\alpha_{\text {Rot }}^{\Gamma 2, R 2 L}$ & $\alpha_{\text {Rot }}^{\Gamma 3, L 2 R}$ & 0.5 \\
\hline Slave & $\alpha_{\text {Trans }}^{\Gamma 1, R 2 L}$ & $\alpha_{\text {Trans }}^{\Gamma 3, R 2 L}$ & 1 \\
\hline Slave & $\alpha_{\text {Rot }}^{I 1, R 2 L}$ & $\alpha_{\text {Rot }}^{\Gamma 3, R 2 L}$ & 1 \\
\hline \multicolumn{4}{|c|}{ TABLE I }
\end{tabular}

TASK Allocation SETtings

\section{EXPERIMENT}

The following experiments have been performed with the DLR HMI - a bimanual haptic device - and the humanoid robot SpaceJustin (see Fig. 12 and Fig. 13). The first two experiments focus the coupling of the master devices by the virtual pipe and the task allocation performance. The third experiment investigates the whole multilateral cooperation involving the slave robot. In all experiments, the time delay has been set to zero for simplification. The track-POPC's robustness against delay has already been shown in previous publications.

In the first experiment, the task allocation is disabled such that master 1 and master 2 have the same authority on the pipe end. Fig. 14 depicts the motion of the pipe. Both devices $\left(P_{m 2}, P_{m 1}\right)$ and thus the virtual pipe are at first moved upwards $(4.5 s$ to $6 s$, see Fig. 15 , the position tracking plots depict position deviations from the initial positions such that the pipe length offset in $y$-direction is

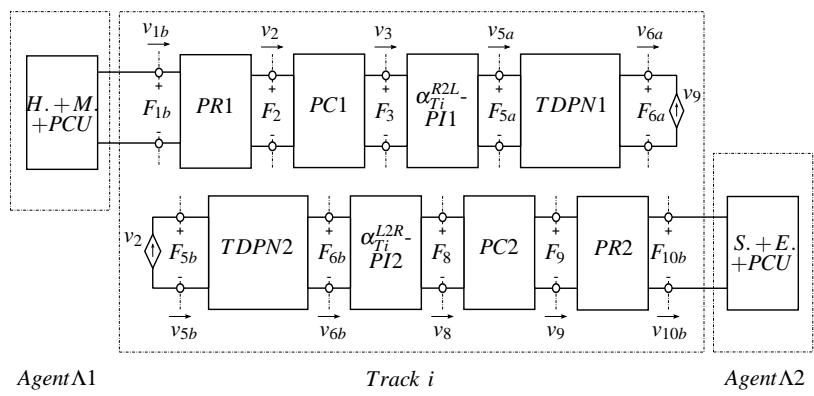

Fig. 11. Network representation of a track-POPC system for cooperative teleoperation with task allocation 


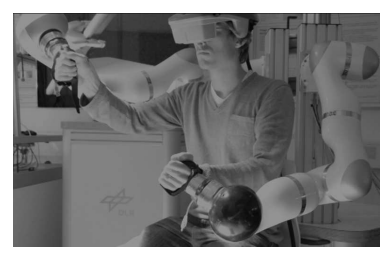

Fig. 12. DLR HMI

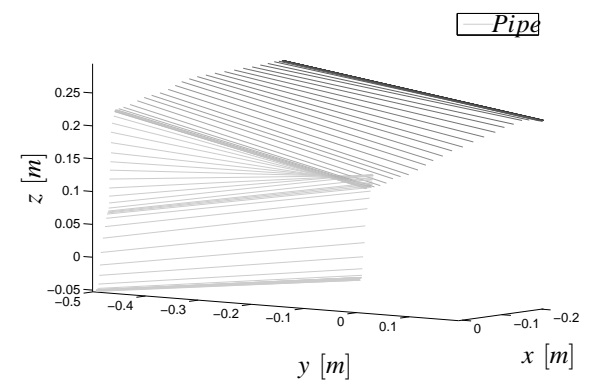

Fig. 14. Pipe motion without task allocation

not considered). Then master 1 reorients the pipe (6s to $7 s$ ) through rotation around $P_{m 2}$ and the virtual gripping point $P_{m 1}^{P E}$, which depends on the master 1 pose $P_{m 1}$, respectively. During the rotation around the pipe end the position of this virtual gripping point is not absolutely fixed, as can be seen in Fig. 14. This happens due to cross couplings of rotations and translations in the robot arms. From second $7 s$ to $10 s$ both devices are moved horizontally. During the whole procedure the master 2 position $P_{m 2}$ and the virtual gripping point $P_{m 1}^{P E}$ match very well.

In the second experiment the task allocation scalings have been chosen corresponding to TABLE I. At first (Fig. 17, $8 s$ to $9.5 s$ ), master 1 rotates the pipe around $P_{m 2}$. Thanks to the task allocation, the operator therefore only needs to keep the rotational DoFs rather loose on both devices and command the rotation around the $x$-axis comfortably and accurately through a force along the z-axis. Then master 2 commands a translational motion of the virtual pipe in the $x y$-plane (see $10 s$ to $12 s$ ). Finally (in the time between
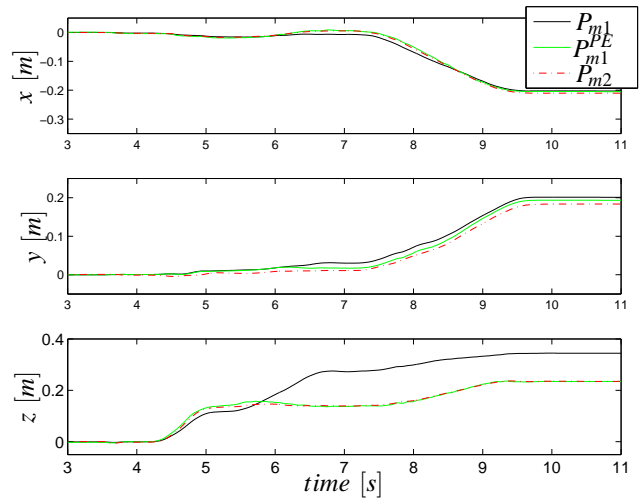

Fig. 15. Position tracking without task allocation

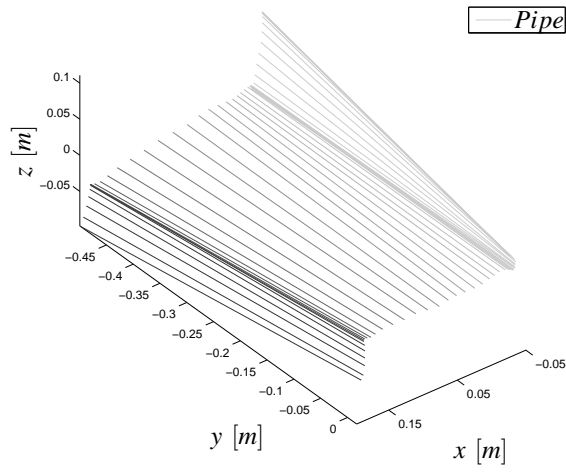

Fig. 16. Pipe motion with task allocation
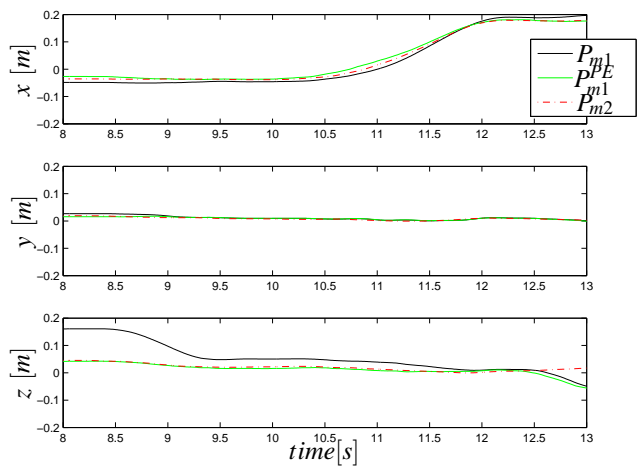

Fig. 17. Position tracking with task allocation

$12.5 s$ to $13 s$ ) master 1 is pushing the virtual pipe down (see Fig. 16), but due to the task allocation, the force part of $W_{m 2}$ acting on master 2 is always zero (compare Fig. 18).

The third experiment involves the whole multilateral cooperation (depicted in Fig. 12 and Fig. 13) with the task allocation values of TABLE I. The task is the insertion of a pipe as depicted in Fig. 19. At first, the real and the virtual pipe are rotated around the end of the real pipe $P_{s}^{P E}$ and the virtual pipe $P_{m 1}^{P E}$ respectively into horizontal orientation. Then the pipe end is pushed to the $x$-position of the plug. In order to plug in the real pipe the devices are afterwards pushed horizontally along the pipe axis ( $y$-axis)
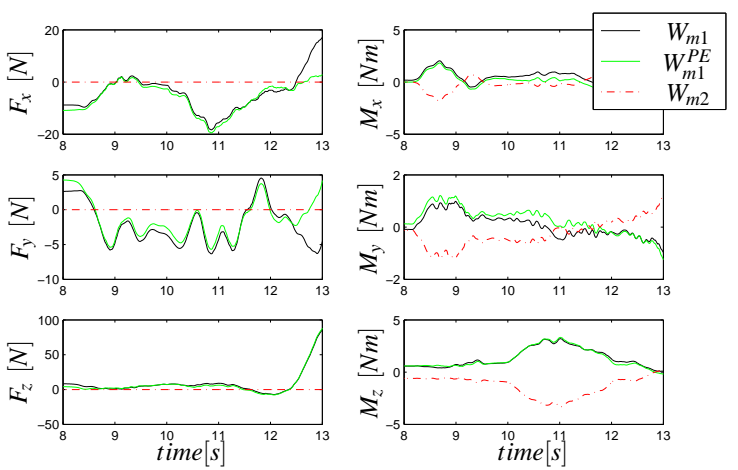

Fig. 18. Forces and torques during manipulation with task allocation 


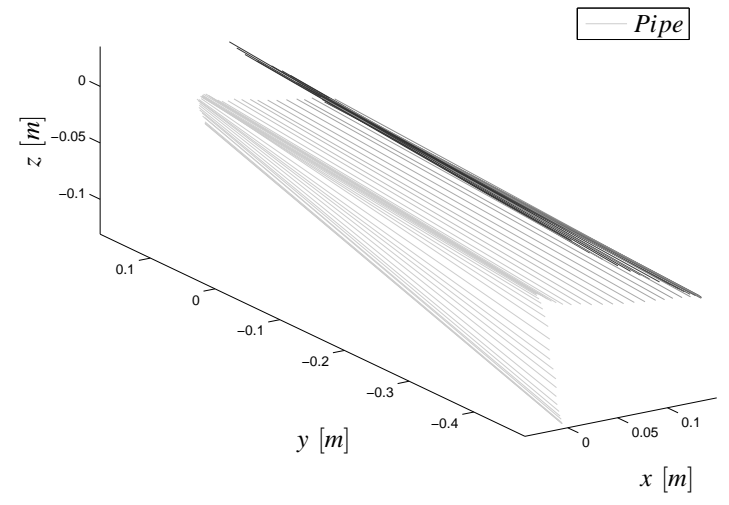

Fig. 19. Pipe motion in multilateral system with task allocation

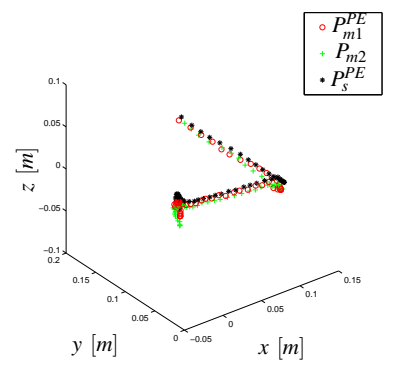

Fig. 20. Tracking of virtual gripping point on real and virtual pipe end and master 2

into the plug. The position following (see Fig. 20 and Fig. 21) of the three devices is absolutely satisfactory though the cross couplings and the constraints caused by singularities in all three robots influence the tracking performance slightly.

\section{CONCLUSION}

The multilateral track-POPC approach for delayed teleoperation has been extended to the multi-DoF case in a MMSS system. A virtual gripping point has been implemented via passive projections to ease the manipulation of a long object by a single slave robot. Through the bimanual operation of the pipe, its rotational DoFs can be more accurately affected through forces than they could be in a unimanual system purely through torques. The cartesian task allocation helped to maintain the congruence of pipe end and plug positions. The position following of the three devices, the virtual and real gripping points was satisfactory.

\section{ACKNOWLEDGMENT}

This paper was supported by the project Development of a Tele-Service Engine and Tele-Robot Systems with MultiLateral Feedback funded by the Ministry of Trade, Industry \& Energy of Korea.

\section{REFERENCES}

[1] D. G. Lee, G. R. Cho, M. S. Lee, B.-S. Kom, S. Oh, and H. I. Son, "Human-centered evaluation of multi-user teleoperation for mobile manipulator in unmanned offshore plants," IEEE International Conference on Intelligent Robots And Systems, pp. 5431-5438, 2013.
[2] A. Peer, S. Hirche, C. Weber, I. Krause, and M. Buss, "Intercontinental multimodal tele-cooperation using a humanoid robot," IEEE International Conference on Intelligent Robots and Systems, pp. 405 - 411, 2008.

[3] S. Sirouspour, "Modeling and control of cooperative teleoperation systems," IEEE Transactions on Robotics, vol. 21, pp. 1220 - 1225, 2005.

[4] C. Passenberg, A. Peer, and M. Buss, "Model-mediated teleoperation for multi-operator multi-robot systems," IEEE International Conference on Intelligent Robots and Systems, pp. 4263 - 4268, 2010.

[5] P. Malysz and S. Sirouspour, "Dual-master teleoperation control of kinematically redundant robotic slave manipulators," IEEE International Conference on Intelligent Robots And Systems, pp. 5115 - 5120, 2009.

[6] M. Panzirsch, J. Artigas, A. Tobergte, P. Kotyczka, C. Preusche, A. Albu-Schaeffer, and G. Hirzinger, "A peer-to-peer trilateral passivity control for delayed collaborative teleoperation," EuroHaptics, vol. 12, pp. 395-406, 2012.

[7] D. Feth, B. An Tran, R. Groten, A. Peer, and M. Buss, "Shared-control paradigms in multi-operator-single-robot teleoperation," in Human Centered Robot Systems. Springer Berlin Heidelberg, 2009, pp. 5362.

[8] S. Sirouspour and P. Setoodeh, "Adaptive nonlinear teleoperation control in multi-master/multi-slave environments," IEEE International Conference on Control Applications, pp. 1263 - 1268, 2005.

[9] T. Kanno and Y. Yokokohji, "Multilateral teleoperation control over time-delayed computer networks using wave variables," Haptics Symposium, pp. 125-131, 2012.

[10] M. Panzirsch, J. Artigas, J.-H. Ryu, and M. Ferre, "Multilateral control for delayed teleoperation," IEEE International Conference on Advanced Robotics, pp. 1-6, 2013.

[11] P. Malysz and S. Sirouspour, "Trilateral teleoperation control of kinematically redundant robotic manipulators," The International Journal of Robotics Research, vol. 30, pp. 1643-1664, 2011.

[12] P. Malysz and S. Sirouspour, "Cooperative teleoperation control with projective force mappings," IEEE Haptics Symposium, pp. 301 - 308, 2010.

[13] S. Katsura, T. Suzuyama, and K. Ohishi, "A realization of multilateral force feedback control for cooperative motion," IEEE Transactions on Industrial Electronics, vol. 54, pp. 3298 - 3306, 2007.

[14] P. Malysz and S. Sirouspour, "Task performance evaluation of asymmetric semiautonomous teleoperation of mobile twin-arm robotic manipulators," IEEE Transactions on Haptics, vol. 6, pp. $484-495$, 2013.

[15] H. V. Quang and J.-H. Ryu, "Stable multilateral teleoperation with time domain passivity approach," IEEE International Conference on Intelligent Robots And Systems, pp. 5890 - 5895, 2013.

[16] H. LeBlanc, E. Eyisi, N. Kottenstette, X. Koutsoukos, and J. Sztipanovits, "A passivity-based approach to deployment in multi-agent networks," in Informatics in Control, Automation and Robotics. Springer Berlin Heidelberg, 2011, pp. 135-149.

[17] N. Y. Chong, T. Kotoku, K. Ohba, and K. Komoriya, "Remote coordinated controls in multiple telerobot cooperation," IEEE International Conference on Robotics And Automation, pp. 3138 - 3143, 2000.

[18] D. Lee and M. W. Spong, "Bilateral teleoperation of multiple cooperative robots over delayed communication network: Theory," IEEE International Conference on Robotics And Automation, pp. 360 - 365, 2005.

[19] R. Mohajerpoor, I. Sharifi, H. A. Talebi, and S. M. Rezaei, "Adaptive bilateral teleoperation of an unknown object handled by multiple robots under unknown communication delay," IEEE International Conference on Advanced Intelligent Mechatronics, pp. 1158 - 1163, 2013.

[20] J. Artigas, J.-H. Ryu, and C. Preusche, "Time domain passivity control for position-position teleoperation architectures," Presence: Teleoperators and Virtual Environments, vol. 19, no. 5, pp. 482-497, 2010.

[21] J. Artigas, J.-H. Ryu, C. Preusche, and G. Hirzinger, "Network representation and passivity of delayed teleoperation systems," IEEE International Conference on Intelligent Robots And Systems, pp. 177183, 2011. 